\title{
BMJ Open Phenotypes of atrial fibrillation diagnosed before-versus-after ischaemic stroke and TIA: study protocol for a systematic review and meta-analysis
}

\author{
Amado Jimenez-Ruiz (D) , ${ }^{1}$ Juan Camilo Vargas-Gonzalez, ${ }^{2}$ Sebastian Fridman, ${ }^{2}$ \\ Luciano A Sposato (iD ${ }^{2}$
}

To cite: Jimenez-Ruiz A, Vargas-Gonzalez JC, Fridman S, et al. Phenotypes of atrial fibrillation diagnosed beforeversus-after ischaemic stroke and TIA: study protocol for a systematic review and meta-analysis. BMJ Open 2021;11:e044288. doi:10.1136/ bmjopen-2020-044288

- Prepublication history for this paper is available online. To view these files, please visit the journal online (http://dx.doi. org/10.1136/bmjopen-2020044288).

Received 29 August 2020 Accepted 20 April 2021

Check for updates

(C) Author(s) (or their employer(s)) 2021. Re-use permitted under CC BY-NC. No commercial re-use. See rights and permissions. Published by BMJ.

${ }^{1}$ Heart \& Brain Lab, Western University Schulich School of Medicine \& Dentistry, London, Ontario, Canada

${ }^{2}$ Clinical Neurological Sciences, London Health Sciences Centre, London, Ontario, Canada

Correspondence to

Dr Amado Jimenez-Ruiz;

dr.amadojimenez@gmail.com

\section{ABSTRACT}

Introduction The underlying pathophysiology of atrial fibrillation (AF) detected after stroke (AFDAS) is relatively unknown. Preliminary evidence suggests AFDAS has a lower prevalence of cardiovascular comorbidities and higher incidence of insular cortex involvement than AF known to exist before stroke occurrence (KAF). This favours a neurogenic AF substrate (autonomic dysregulation) in which the presence of underlying heart disease is not necessary for AF to occur. The main objective of this systematic review and meta-analysis is to compare the prevalence of cardiovascular comorbidities and echocardiographic abnormalities in patients with AFDAS, KAF and no AF (NAF). Secondary objectives are to compare the proportion with insular cortex involvement, stroke recurrence and death in the three rhythm groups. Methods and analysis We will perform a systematic review including cross-sectional, case-control, cohort studies and clinical trials involving $\geq 18$ years patients, with ischaemic stroke or transient ischaemic attack published between inception and 31 December 2020 in any language, and reporting the proportion of patients with AFDAS, KAF and NAF. We will search PubMed, EMBASE and Scopus by applying predefined search terms. Two reviewers will independently screen titles and abstracts and retrieve full texts, extract data in a predesigned form, and assess the risk of bias. We will perform a meta-analysis of all included studies and we will report the results of the main outcome as proportions. We will report results of secondary outcomes as risk ORs. We will estimate heterogeneity across studies by using $\mathrm{t}^{2}, \mathrm{Q}$ and $\mathrm{I}^{2}$ measures. We will use funnel plots, Rosenthal's Fail-Safe $\mathrm{N}$ and Egger's regression intercept to assess publication bias.

Ethics and dissemination This study will be based on published data and does therefore not require ethical clearance. The results will be published in peer-reviewed journals.

PROSPERO registration number CRD42020202622.

\section{INTRODUCTION}

Incident atrial fibrillation (AF) can be diagnosed in up to $24 \%$ of patients with ischaemic stroke or transient ischaemic attack (TIA). ${ }^{1}$ It has been hypothesised that AF detected
Strengths and limitations of this study

- This study will systematically compare clinical phenotypes of patients with incident atrial fibrillation (AF) after ischaemic stroke or transient ischaemic attack, those with prevalent $A F$, and those with no AF.

- We will apply well-validated systematic review and meta-analysis tools that are fully compliant with current international guidelines and recommendations.

- A limitation of this study is that we anticipate that there will be different methods used for AF detection and duration of monitoring across cohorts may vary, resulting in some heterogeneity.

- The number of studies comparing stroke recurrence and death (secondary outcomes of this study) in the prespecified groups may be low, limiting the possibility of performing a reliable meta-analysis.

- Reporting of other variables needed for secondary analyses (eg, involvement of the insular cortex) may be also low, limiting the feasibility of the proposed analyses.

after stroke (AFDAS) has distinctive pathophysiology involving autonomic dysfunction and inflammation ${ }^{2}$ as well as specific clinical characteristics and outcomes relative to AF known before stroke occurrence (KAF). ${ }^{3}$ AFDAS comprises neurogenically triggered $\mathrm{AF}$ in patients with structurally normal hearts and no underlying cardiovascular comorbidities, while $\mathrm{KAF}$ is the classically known $\mathrm{AF}$ in patients who are unaware of the arrhythmia until they experience a cerebrovascular event. ${ }^{3}$ Based on non-systematically gathered data, it has been previously hypothesised that AFDAS is a relatively benign type of AF compared with $\mathrm{KAF}^{2}$ because of being low burden, ${ }^{4}$ associated with lower prevalence of cardiovascular comorbidities, ${ }^{5}$ and lower risk of stroke recurrence. ${ }^{6}$ To the best of our knowledge, only three studies have evaluated differences between patients with AFDAS, $\mathrm{KAF}$ and no AF (NAF) ${ }^{6-8}$ All these studies 
found a lower prevalence of cardiovascular comorbidities and lower $\mathrm{CHA}_{2} \mathrm{DS}_{2}$-VASc score in patients with AFDAS compared with those with KAF. Additionally, one of these studies showed that patients with AFDAS have lower recurrence rates of ischaemic stroke. ${ }^{6}$ Although the results of these studies favour our hypothesis, they require validation through a thorough and comprehensive systematic search and meta-analysis. We will, therefore, perform a systematic review and meta-analysis of studies reporting the prevalence cardiovascular comorbidities, structural heart disease, insular involvement, recurrent stroke and death.

\section{Review questions}

- Are pre-existing cardiovascular comorbidities and echocardiographic abnormalities less prevalent in AFDAS than KAF patients, and not significantly more prevalent than in patients with NAF?

- Are there differences in the prevalence of insular cortex involvement in patients with AFDAS, KAF and NAF?

- What is the risk of stroke recurrence at 1 year after stroke in patients with AFDAS, KAF and NAF?

- What is the risk of in-hospital death and death at 30 days and 1 year after stroke in patients with AFDAS, $\mathrm{KAF}$ and NAF?

\section{Objectives}

- Primary objective: To compare the prevalence of cardiovascular comorbidities (coronary artery disease, heart failure, AF) and echocardiographically evident structural heart disease (either left atrial enlargement or decreased left ventricular ejection fraction) in patients with AFDAS, KAF and NAF.

- Secondary objective: (1) To compare the incidence of insular cortex involvement in patients with AFDAS, KAF and NAF; (2) To compare the risk of stroke recurrence at 1 year between AFDAS, KAF and NAF and (3) to compare the risk of in-hospital death and death at 30 days and 1 year between AFDAS, KAF and NAF.

\section{METHODS}

This study protocol has been prepared according to the 2015 Preferred Reporting Items for Systematic Reviews and Meta-Analyses Protocols guidelines. ${ }^{4}$

\section{Criteria for considering studies for the review Inclusion criteria}

We will include all cross-sectional, case-control, cohort studies and clinical trials published inception and 31 December 2020 in any language involving adults (18 years of age or older) and reporting the prevalence of patients with AFDAS, KAF or NAF.

\section{Exclusion criteria}

We will exclude reviews, letters to the editor, editorials, conference articles with incomplete data, studies with a small sample size (less than 30 participants). For duplicated publications (reports including the same

\section{Box 1 PubMed search terms}

(stroke[(MeSH Terms])) AND (atrial fibrillation[(MeSH Terms])) (ischemic attack, transient[(MeSH Terms])) AND (atrial fibrillation[(MeSH Terms]))

(brain ischemia[(MeSH Terms])) AND (atrial fibrillation[(MeSH Terms])) (stroke[(MeSH Terms])) AND (Holter[(Tiab]))

(ischemic attack, transient[(MeSH Terms])) AND (Holter[(Tiab]))

(brain ischemia[(MeSH Terms])) AND (Holter[(Tiab]))

(brain ischemia[(MeSH Terms])) AND (electrocardio*[(Tiab]))

(stroke[(MeSH Terms])) AND (electrocardio*[(Tiab]))

(ischemic, transient[(MeSH Terms])) AND (electrocardio*[(Tiab]))

(ischemic attack, transient[(MeSH Terms])) AND (telemet*[(Tiab]))

(stroke[(MeSH Terms])) AND (telemet*[(Tiab]))

(brain ischemia[(MeSH Terms])) AND (telemet*[(Tiab]))

(brain ischemia[(MeSH Terms])) AND (mobile*[(Tiab]))

(ischem attack, transient[(MeSH Terms])) AND (mobile*[(Tiab]))

(stroke[(MeSH Terms])) AND (mobile*[(Tiab]))

(stroke[(MeSH Terms])) AND (MCOT*[(Tiab]))

(brain ischemia[(MeSH Terms])) AND (MCOT*[(Tiab]))

(ischemic attack, transient[(MeSH Terms])) AND (MCOT*[(Tiab]))

stroke [(Ti]) AND atrial fibrillation[(Ti])

population), we will collate multiple reports to craft the most comprehensive database from that study.

\section{Search strategy for the identification of relevant studies}

We will search PubMed, EMBASE and Scopus to identify potentially eligible studies by applying predefined search terms. Search terms are shown in boxes 1-3. We will also use the 'similar articles' PubMed function (first 50 articles listed per article included in the study), we will screen the reference lists of included articles and we will search each of this study authors' personal archives for additional relevant publications that were not identified in the study search.

\section{Box 2 EMBASE search terms}

heart atrium fibrillation[(Map Term]) AND cerebrovascular accident[(Map Term])

heart atrium fibrillation[(Map Term]) AND transient ischemic attack[(Map Term])

(cerebrovascular accident[(Map Term])) AND (Holter monitoring or Holter monitor[(Map Term]))

(transient ischemic attack[(Map Term])) AND (Holter monitoring or Holter monitor[(Map Term]))

(cerebrovascular accident[(Map Term])) AND (electrocardiography or electrocardiogramECG[(Map Term]))

(transient ischemic attack[(Map Term])) AND (electrocardiography or electrocardiogramECG[(Map Term]))

(cerebrovascular accident[(Map Term])) AND (telephone telemetry or telemetry[(Map Term]))

(transient ischemic attack[(Map Term])) AND (telephone telemetry or telemetry[(Map Term]))

(cerebrovascular accident[(Map Term])) AND (Mobile[(Map Term]))

(transient ischemic attack[(Map Term])) AND (Mobile[(Map Term])) 


\section{Box 3 Scopus search terms}

Atrial fibrillation AND stroke [Title, abstract, keyword]; subject area: health sciences; document type: article

\section{Selection of studies for inclusion in the review}

Two reviewers will independently screen titles and abstracts by using COVIDENCE and will solve disagreements by consensus (LAS and AJ-R). In cases of persisting disagreement, a third reviewer will intervene (JCV-G). The same reviewers will fully assess all potentially relevant records. We will document reasons for excluding specific publications.

\section{Assessment of the methodological quality and risk of bias}

To evaluate the methodological quality and risk of bias of each publication, we will use the risk of bias in nonrandomised studies of interventions (ROBINS-I) ${ }^{5}$ on six domains: confounding, participants' selection bias, deviations from intended interventions, missing data, ascertainment of outcomes, and results reporting bias. We will classify the results following the ROBINS-I criteria as low, moderate, serious, critical risk of bias or no information. ${ }^{5}$

\section{Data extraction and management}

We will create and use a standardised COVIDENCE data extraction form including the following.

- Study identification: funding source, country, setting, author name, institution, email, address and possible conflicts of interest.

- Study characteristics: study design, groups, aim of the study, start date, inclusion and exclusion criteria, recruitment methods and setting.

- Patients' characteristics:

All patients with ischaemic stroke with or without prior AF diagnosis. Selected papers will be those in which the results of cardiac monitoring $\geq 24$ hours are reported and include information about any of the following five outcomes:

1. Cardiovascular comorbidities defined as coronary artery disease, heart failure, prior myocardial infarction.

2. Echocardiographic parameters defined as wall motion abnormalities, decreased ejection fraction or dilated left atrium.

3. Proportion with involvement of the insular cortex.

4. Stroke recurrence at 1 year.

5. Death rate the time of discharge, at 30 days and at 1 year.

Other variables to be recorded include: mean or median age (years), mean or median National Institutes of Health stroke scale (points), elevated cardiac troponin $(\mathrm{n})$, hypertension $(\mathrm{n})$, diabetes mellitus (n), chronic kidney disease $(n)$, dyslipidaemia $(n)$, active smoking $(n)$, alcohol misuse $(n)$, prior ischaemic stroke $(n)$, prior TIA (\%), prior intracerebral hemorrhage (ICH) (n), dementia (n), Trial of ORG 10172 in Acute Stroke
Treatment category, embolic stroke of undetermined source and brain infarct/volume (mL).

- Main exposure: The main exposure will be AFDAS and will be used as the reference group. KAF and NAF will be compared against AFDAS.

\section{Data analysis and reporting}

We will apply random-effects or fixed-effects metaanalyses depending on the source of heterogeneity to estimate the proportion stroke patients in each of the three heart rhythm groups with prevalent cardiovascular comorbidities and echocardiographic abnormalities. For the secondary objectives, except for the prevalence of insular cortex involvement, we will report risk ratios or ORs. We will use the Agresti-Cuoll method to calculate CIs for individual studies. We will calculate variance tau between studies with the maximum-likelihood estimator.

We will estimate heterogeneity across studies by using $\mathrm{t}^{2}, \mathrm{Q}$ and $\mathrm{I}^{2}$ measures. We will perform subgroup analyses to account for heterogeneity between types of stroke. Additionally, we will use the 'leave-one-out' procedure as a sensitivity analysis to identify studies responsible for heterogeneity for each stroke type ${ }^{6}$. We will perform a combinatorial meta-analysis and we will apply a graphical display of study heterogeneity $(\mathrm{GOSH}) .{ }^{7}$ If outliers are found, we will use colour-code subgroup meta-analyses with and without the outlier study to enhance the GOSH plot.

We will perform a meta-regression using the randomeffects model if data allows for exploring the different continuous acute myocardial injury (AMI) and chronic myocardial injury (CMI) variables.

We will evaluate whether selective reporting of outcomes is present. We will compare the fixed effect estimate against the random effects model to assess the possible presence of small sample bias in the published literature. We will apply enhanced funnel plots, Rosenthal's FailSafe N, Duval and Tweedie's trim and fill procedure, and Egger's regression intercept for evaluating reporting bias if at least 10 studies are retrieved.

We will conduct all analyses with R V.3.6.2 (R Core Team, 2014), by using the 'Meta' and 'Metaphor' packages according to the Cochrane Handbook for systematic reviews.

\section{Patient and public involvement statement}

No patient was involved in the planning or design of the study.

\section{Potential amendments}

We do not anticipate any amendment to this review protocol. However, if amendments are needed, they will be documented and reported transparently.

\section{Ethics and dissemination}

This systematic review and meta-analysis will be based on published data. Thus, it does not require specific ethical approval. The results will be published in peer-reviewed journals and presented at scientific conferences. 
Twitter Luciano A Sposato @SposatoL

Contributors LAS conceived the study. AJ-R and LAS drafted the manuscript. LAS JCV-G and AJ-R revised the manuscript. All authors approved the final version. LAS is the guarantor of the review.

Funding LAS is supported by the Kathleen \& Dr Henry Barnett Research Chair in Stroke Research (Western University, London, Canada); the Edward and Alma Saraydar Neurosciences Fund (London Health Sciences Foundation, London, Canada); and the Opportunities Fund of the Academic Health Sciences Centre Alternative Funding Plan of the Academic Medical Organization of Southwestern Ontario (AMOSO) (Ontario, Canada).

Competing interests LAS speaker and consulting honoraria from Boehringer Ingelheim, Pfizer, Bayer and Gore; research grants from Boehringer Ingelheim and Bayer. LAS is member of the Editorial Board of the Neurology and Stroke journals, and the World Stroke Academy (Webinar platform of the World Stroke Organization). The remaining authors have nothing to disclose.

Patient and public involvement Patients and/or the public were not involved in the design, or conduct, or reporting, or dissemination plans of this research.

Patient consent for publication Not required.

Provenance and peer review Not commissioned; externally peer reviewed.

Open access This is an open access article distributed in accordance with the Creative Commons Attribution Non Commercial (CC BY-NC 4.0) license, which permits others to distribute, remix, adapt, build upon this work noncommercially, and license their derivative works on different terms, provided the original work is properly cited, appropriate credit is given, any changes made indicated, and the use is non-commercial. See: http://creativecommons.org/ licenses/by-nc/4.0/.
ORCID iDs

Amado Jimenez-Ruiz http://orcid.org/0000-0002-0257-3107

Luciano A Sposato http://orcid.org/0000-0001-6425-9343

\section{REFERENCES}

1 Sposato LA, Cipriano LE, Saposnik G, et al. Diagnosis of atrial fibrillation after stroke and transient ischaemic attack: a systematic review and meta-analysis. Lancet Neurol 2015;14:377-87.

2 Sposato LA, Hilz MJ, Aspberg S, et al. Post-Stroke cardiovascular complications and neurogenic cardiac injury: JACC state-of-the-art review. J Am Coll Cardiol 2020;76:2768-85.

3 Cerasuolo JO, Cipriano LE, Sposato LA. The complexity of atrial fibrillation newly diagnosed after ischemic stroke and transient ischemic attack: advances and uncertainties. Curr Opin Neurol 2017;30:28-37.

4 Sposato LA, Cipriano LE, Riccio PM, et al. Very short paroxysms account for more than half of the cases of atrial fibrillation detected after stroke and TIA: a systematic review and meta-analysis. Int $J$ Stroke 2015;10:801-7.

5 Sposato LA, Seiffge DJ. Atrial fibrillation detected after stroke and increased risk of death. Neurology 2021;96:557-9.

6 Sposato LA, Cerasuolo JO, Cipriano LE, et al. Atrial fibrillation detected after stroke is related to a low risk of ischemic stroke recurrence. Neurology 2018;90:e924-31.

7 Hsieh C-Y, Lee C-H, Wu DP, et al. Characteristics and outcomes of ischemic stroke in patients with known atrial fibrillation or atrial fibrillation diagnosed after stroke. Int J Cardiol 2018;261:68-72.

8 Yang X-M, Rao Z-Z, Gu H-Q, et al. Atrial fibrillation known before or detected after stroke share similar risk of ischemic stroke recurrence and death. Stroke 2019;50:1124-9. 